\title{
Removal of azithromycin from aqueous bulk solution using water/ethanol/transcutol/capryol-90 green nanoemulsion
}

Afzal Hussain ( $\square$ afzal.pharma@gmail.com )

King Saud University College of Pharmacy

Sultan Alshehri

King Saud University

Mohammad A. Altamimi

King Saud University

Mohd Usman Mohd Siddique

Shri Vile Parle Kelavani Mandal's Institute Of Technology

\section{Research Article}

Keywords: Azithromycin, Green nanoemulsion, Thermodynamic stability, Impact of components and nanoemulsion parameters, \% Removal efficiency

Posted Date: March 31st, 2021

DOl: https://doi.org/10.21203/rs.3.rs-337792/v1

License: (9) This work is licensed under a Creative Commons Attribution 4.0 International License. Read Full License 


\section{Abstract}

The present work aimed to remove azithromycin (AZM) from contaminated aqueous system using a water/ethanol/transcutol/capryol-90 green nanoemulsion. The drug is identified as potential pharmaceutical drug for detrimental effect on flora and fauna of aquatic lives and human health. Green nanoemulsions were tailored and characterized for thermodynamic stability, globular size, polydispersity index (PDI), zeta potential, viscosity, refractive index (RI), and morphological assessment using transmission electron microscopy (TEM). Moreover, nanoemulsions were investigated for percent removal efficiency and factors affecting the \%RE. The results obtained from in vitro characterization parameters suggested that the developed green nanoemulsions (ANE1-ANE5) were stable, and possessing globular size $₫ 200 \mathrm{~nm}$. ANE5 exhibited the lowest value of globular size (49 nm), low PDI (0.17), minimum viscosity ( $93 \mathrm{cP})$, and optimum zeta potential $(-27.8 \mathrm{mV})$. The value of \%RE depended upon the content of water and capryol-90 oil of the nanoemulsion. Furthermore, the value of \%RE was found to be increased with increased content of water and decreased concentration of capryol-90 in the nanoemulsions. Similarly, \% RE value was increased with decreased value of globular size and decreased viscosity of nanoemulsion. There was no impact of contact time on \%RE results. Thus, the maximum \%RE value (96.8\%) was obtained by ANE5 from the aqueous solution after 20 min of contact time with ANE5. Thus, this method could be a promising approach to remove AZM from a contaminated water and suitable alternative to conventional methods.

\section{Highlights Of The Study}

1. Pharmaceuticals contaminated wastewater challenged human health and aquatic lives

2. Azithromycin (AZM) is commonly used antibiotic and exposed in water as effluent

3. Nanoemulsion could be a promising approach to remove AZM

\section{Introduction}

Azithromycin (AZM) is one of the most effective and efficient macrolide based broad spectrum antibiotics used to control several bacterial infections. AZM is one of the most prominent and emerging contaminant among pharmaceutical compound (Segura et al. 2009). A trace amount of AZM in the aquatic environment raised a serious concern due to chemical stability, high biological activity, and constant accumulation to toxic level which results in causing adverse effect to non-targeted species including human (Fent et al. 2006; Segura et al. 2009). Avila et al. reported AZM as the second antibiotic (among 13 selected antibiotics) at the highest level (379-625 ng/L) in influent wastewater of constructed wetland (Avila et al. 2021). Moreover, authors achieved about 53\% removal efficiency using a vertical flow wetland system (Avila et al. 2021). Few authors reported removal efficiency (\%) from negative to $86 \%$ for AMZ using UVF unit of plants (Verlicchi and Zambello 2014). As per the Watch list of EU (European Union), 2015, three macrolides (erythromycin, azithromycin, and clarithromycin) are still poorly investigated (15.6\%) on this concern as compared to others (CECs 2015). In last decade, various 
conventional methods (chemical, physical and biological) have been reported to remove AZM from wastewater of different resources such as industrial and municipal wastewater. However, these methods were not much efficient to remove AZM completely from water. The wastewater with low concentration of AZM reduces the efficiency of the biological process of water treatment due to microbial inhibition (Luo et al. 2016). Luo et al. assessed photocatalytic degradation method of wastewater treatment containing low concentration of AZM. They observed that the removal efficiency of AZM was greatly influenced with the $\mathrm{pH}$, the size of activated carbon, reaction time and different La-TiO2 mass ratio (Luo et al. 2016). Moreover, these reported methods were either complex or expensive to set up for large scale processing. In 2015, a low cost modified form of Fenton reaction was utilized to eliminate AZM from the wastewater treatment plant and the result $(919 \pm 140 \mathrm{ng} / \mathrm{L})$ could not convince after analysis of the effluent water (Mackul'ak et al. 2015). Very recently, low frequency ultrasound (40 kHZ) with or without hydrogen peroxide in aqueous solution was used to remove AZM. Authors studied the effect of solution $\mathrm{pH}(3-9), \mathrm{Fe}$ ion, and UV light for the removal of AZM from aqueous solution using ultrasound (Muñoz-Calderón, et al. 2020). The test was conducted at laboratory using an aqueous solution $(1 \mathrm{mg} / \mathrm{L})$ for $1 \mathrm{~h}$ in a reaction volume of $300 \mathrm{~mL}$. The removal efficiency was greatly influenced with the external operational parameters such as $\mathrm{pH}$, ultrasound, presence of ferrous ion, hydrogen peroxide and UV light. However, the achieved \%RE was approximately 50 \% only (Muñoz-Calderón, et al. 2020). Furthermore, macrolides (erythromycin, azithromycin and clarithromyci) removal efficiencies were relatively lower as compared to fluoroquinolones (ciprofloxacin, ofloxacin and norfloxacin) from the influent of sewage wastewater collected from different countries (Ghosh et al. 2016). No authors reported to remove potentially toxic AZM from contaminated water using a green nanoemulsion method so far. Nanoemulsions are thermodynamically stable, cost effective, simple and scalable for large scale processing plant. Additionally, this is an isotropic system comprised of surfactant, co-surfactant, lipid and water with effective solubilizing and extraction capacity (Shakeel et al. 2014a). Several reports have been published to remove pharmaceuticals (drugs) or dyes using green nanoemulsions such as glibenclamide, indomethacine, clarithromycin and methylene blue (Shakeel et al. 2014a; Shakeel et al. 2014b; Shakeel et al. 2015; Mahdi et al. 2021). Excipients were selected based solubility profile of AZM. Then, various nanoemulsions were constructed as per pseudo-ternary phase. The best ratio of surfactant to cosurfactant was chosen based on the maximum zone of nanoemulsion delineated in phase diagrams. The prepared nanoemulsions were assessed for thermal stability followed by characterizations (particle size, polydispersity index, zeta potential, viscosity, and refractive index). The removal efficiency is greatly affected with the content of oil phase and water content. Therefore, we investigated the effect of several potential factors affecting the removal efficiency of AZM from an aqueous solution at lab scale.

\section{Materials And Methods}

\section{Materials}

Azithromycin (AZM, $\geq 98 \%$ pure) was procured from Wockhardt (Aurangabad, India). Transcutol-HP (THP, Diethylene glycol monoethyl ether) was obtained from Gattefosse (France). Capryol-90 (MG-90) and capmul MCM C8 (CMC8, capric and caprylic acids) were gifted from ABITEC (Janesville, Germany). 
Ethanol, methanol, tween 80, Propylene glycol, polyethylene glycol, acetonitrile, and buffers were procured from Sigma-Aldrich, Mumbai, India. All other reagents were of analytical grade. Millipore water was used an aqueous solvent.

\section{Methods}

\section{Identification of the drug: Differential Scanning Calorimeter (DSC)}

Thermal analysis was conducted using a DSC technique to estimate fusion temperature (melting point) and enthalpy value. A weighed amount of AZM (3-4 mg) was transferred into a pan and placed inside a furnace using a sample holder. The sample was processed with heating rate of $10^{\circ} \mathrm{C} / \mathrm{min}$ over the temperature range of $10-200^{\circ} \mathrm{C}$.

\section{Solubility assessment in various excipients}

The solubility of AZM was determined in various surfactant, co-surfactant and oil before selecting components of green nanoemulsion. Weighed amount of each excipient was transferred to a clear glass vial and the drug was added to it till saturation. The study was carried out using a water shaker bath (Remi Equipment, Mumbai India) maintained at $40 \pm 1^{\circ} \mathrm{C}$ for $24 \mathrm{~h}$. After completion of $24 \mathrm{~h}$, the mixture was centrifuged and the supernatant was removed followed by filtering with a syringe membrane filter (Whatman, USA). The filtered supernatant was diluted in methanol before analysis. The content of AZM solubilized was estimated using a validated UPLC method at $\lambda_{\max }$ of $483 \mathrm{~nm}$ (Davoodi et al. 2015; Assi et al. 2020). The study was replicated to get mean and standard deviation.

\section{Construction of pseudo ternary phase diagrams and nanoemulsions}

It was prerequisite to select a proper proportion of surfactant and co-surfactant before tailoring a stable and transparent nanoemulsion. This was carried out to identify nanoemulsion zone where the total content of three components (oil, water and $\mathrm{S}_{\text {mix }}$ ) was $100 \%$. Capryol-90 (MG-90) as oil, THP as surfactant and ethanol as co-surfactant were selected based on the maximum solubility of AZM at $40{ }^{\circ} \mathrm{C}$. For this, a series of nanoemulsions were prepared by titrating the oil and selected $S_{\text {mix }}$ ratios $(1: 1,1: 2,1: 3$, and 2:1) in different volume ratio (1:9 to 9:1). The nanoemulsions were prepared by slow titration and emulsification method (Shakeel et al. 2015). These nanoemulsions were placed overnight at room temperature to observe any sign of instability. Those nanoemulsions covering maximum region (delineated area) in pseudo ternary phase diagram were selected for further studies and characterization parameters.

\section{Freeze-thaw and centrifugation stability study: Thermodynamic stability study}

Nanoemulsions were prepared and subjected for thermodynamic stability and centrifugation (physical stress). All nanoemulsions (ANE1-ANE5) were exposed to the cycles of three temperatures $\left(-21^{\circ} \mathrm{C}, 4^{\circ} \mathrm{C}\right.$, and $45^{\circ} \mathrm{C}$ ) followed by ultracentrifugation (coulter counter, Beckman, USA) at $36288 \times \mathrm{g}$ for $30 \mathrm{~min}$ (Khalil et al. 2021). In brief, the samples (5 mL) in the glass vials were stored at three different cycles of 
temperature and centrifugation. In first step (in freeze thaw cycles), green nanoemulsions were kept in a deep freezer at $-21^{\circ} \mathrm{C}$ for $24 \mathrm{~h}$. Then, they were removed and kept at room temperature to return in previous stable form in $<5 \mathrm{~min}$. They were kept undisturbed and inspected for any signs of instability (creaming, turbidity and phase separation). In step 2, the nanoemulsions stable from step 1, were subjected for ultracentrifugation (three cycles repeated). For this, the samples $(2 \mathrm{~mL})$ were transferred to a sample holder tube and exposed to high centrifugation force by ultra-centrifugation for $5 \mathrm{~min}$. Again, the samples were visualized for separation of phase or creaming or cracking. In step 3, six cycles of heating cooling cycle were performed between two temperatures $\left(4^{\circ} \mathrm{C}\right.$ and $\left.40^{\circ} \mathrm{C}\right)$ by storing the nanoemulsions passed after step 2 at $48 \mathrm{~h}$ (Ali et al. 2014). Nanoemulsions after passing step 3, are eligible for further studies.

\section{Characterization of ANE1-ANE5 nanoemulsions}

All of the nanoemulsions were characterized for globular size, polydispersity index (PDI), zeta potential, viscosity, refractive index (REFI) and morphological behavior.

\section{Globular size and size distribution (PDI)}

All of the nanoemulsion passing the thermodynamic stability test, were used for globular size and size distribution. Globular size and PDI were evaluated using a zetasizer (Malvern Nano ZS-90 zetasizer, Worcestershire, UK). Each nanoemulsion was diluted (100 times) using Millipore water before size analysis. The analysis of particle size analyzer works on the principle of "diffraction light scattering (DLS)". The technique is based on the measurement of the intensity of light scattered as a beam of light passes through a dispersed globules or particles in bulk medium. PDI indicates the size distribution of globules in dispersed medium. The analysis was carried out at ambient temperature and scattering angle of $90^{\circ}$. The experiment was performed in triplicate. The average mean size $(Z)$ is calculated using equation 1:

$$
\mathrm{Z} \text { - average globular mean size }(\mathrm{Dz})=\left[\frac{\Sigma(S i)}{\Sigma\left(\frac{S i}{D i}\right)}\right]
$$

where Si and $\mathrm{Di}$ are the scattered light intensity of the particle $i$ and the hydrodynamic diameter of the particle $i$, respectively. The average globular mean size was calculated based on "the intensity based harmonic mean". There may be a slight difference in the particle size assessed by the DLS method as compared to transmission electron microscopy (TEM). This difference is generated due to instrumental error and reported as "fold error" which should be less than 2 for acceptability. The fold error is estimated using the equation 2 (Hussain et al. 2016; Luo et al. 2020):

Fold error $(\mathrm{FE})=10^{1 / \mathrm{n} \times \sum \log (\text { Size, zetasizer } / \text { size, TEM) }}$ 
Where " $n$ " is the experimental time points used for repetition.

Zeta potential values of nanoemulsions were estimated using the same instrument with dilution of the samples. The values of zeta potential may be zero, negative and positive. It represents the surface charge density due to chemical nature of lipid or oil or fatty acids. In general, lipid possesses negative zeta potential due to fatty acid and presence of carboxylic group. Presence of similar charge on the nanoglobules of nanoemulsion results in repulsion and subsequently stability of nanoemulsion. Therefore, dilution was not performed before analysis of the sample at ambient temperature. The experiments were replicated for mean and standard deviation.

\section{Viscosity assessment}

The developed nanoemulsions were isotropic, transparent and thermodynamically stable. Therefore, rheological property was required to determine its flow property upon mixing with water. The viscosity of all nanoemulsions were determined using a Bohlin viscometer (Bohlin Visco 88, Malvern, UK) at room temperature $\left(25^{\circ} \mathrm{C}\right)$ (Hussain et al. 2016). The viscosity was measured at a shear rate from 0 to $100 \mathrm{~s}^{-1}$. The cone and plate were coaxially arranged vertically to the roof of viscometer. The required sample was placed on the plate and cone was allowed to low down over the plate. The sample was processed for a given time period till completion of cycles (ascending and descending cycles). The experiment was repeated for mean and standard value. Generally, oil in water $(\mathrm{o} / \mathrm{w})$ nanoemulsion is relatively low viscous as compared to water in oil (w/o) type of nanoemulsion. This is due to water as continuous phase in former case (o/w) (Mahdi et al. 2021).

\section{Refractive index (RI) of oil and green nanoemulsions}

Nanoemulsions are transparent and isotropic biphasic system with thermodynamic stability. Refractive index is an optical property which can be assessed for these nanoemulsions. The RI values were determined for all ANF1-ANF5 using an Abbe type refractometer (Bausch and Lomb Optical Company, Rochester, New York, USA). Millipore water and capryl-90 were also used for RI assessment. Water served as control for comparison against ANF1-ANF5 nanoemulsions. The sample (one drop) was kept on the slide and processed at $25^{\circ} \mathrm{C}$ (Ali et al. 2014). As per "effective medium theory", the refractive index difference is linear dependent on the concentration of the surfactant. Thus, a normalized refractive index difference (dimensionless) " $X$ " is mathematically expressed as

$$
\mathrm{x}=\frac{(n o-n)}{n o}
$$

Where " $n_{0}$ " and " $n$ " are the refractive indexes of the pure solvent (water) and the nanoemulsion, respectively. This model is application for a solution or nanoemulsion system having globular size far below the wavelength of visible light. If the globular size approaches near the wavelength of visible spectrum or beyond the visible spectrum, the equation 3 is not application due to the scattering of light at 
the visible wavelength range. RI depends upon several factors of nanoemulsion or dispersion such as globule shape, size, polydispersity index and particle volume radius (Zhu et al. 2012). For turbid and submicron sized dispersion, it is difficult to measure accurate RI value using Abbe type refractometer since the light-dark boundary becomes blurred due to the scattering of light by the large size particles.

\section{Preparation of stock solution}

AZM is minimal solubility in water at room temperature $(\sim 2.37 \mu \mathrm{g} / \mathrm{mL})$. Moreover, the drug is soluble in ethanol and dimethyl sulfoxide (DMSO). In order to prepare a stock solution, a weighed amount of AMZ was completely dissolved in water containing DMSO $(1 \% \mathrm{v} / \mathrm{v})$. Final strength of the drug solution (stock solution) was $100 \mathrm{ppm}$. A range of concentration was prepared through dilution (0.1-100.0 ppm). The content of the drug was estimated quantitatively using UPLC method at $482 \mathrm{~nm}$ (Assi et al. 2020).

\section{An adsorption study: percent removal efficiency (\%RE)}

AZM is hydrophobic and poorly soluble in water. We had prepared stock solution AMZ in aqueous system. \% RE of AZM from an aqueous solution was carried out by complete dispersion of weighed quantity of nanoemulsion (NE, 1g) in $10 \mathrm{ml}$ of the stock solution $(100 \mathrm{ppm} / 10 \mathrm{~mL})$. The drug solution and NE mixture was rigorously vortexed for 20 min followed by standing at $25^{\circ} \mathrm{C}$. Each nanoemulsion was individually treated with the drug stock solution for 5, 10, and 20 min. Eventually, the mixture was centrifuged at 10,000 rpm for $20 \mathrm{~min}$ to get a supernatant. The supernatant was withdrawn and analyzed for AZM content at each time point. The drug concentration $\left(X_{t}\right)$ at time point " $t$ " was assayed and presented in " $\mathrm{ppm} / \mathrm{g}$ ". The drug content adsorbed onto the NE surface was quantified at 5, 10 and 20 min using equation 1 :

$Q=\left[\left(X_{0}-X_{t}\right) / w\right] \times V$

Thus, \% RE was calculated using equation 2:

$\% R E=\left[\left(X_{0}-X_{t}\right) / X_{0}\right] \times 100$

The " $\mathrm{X}_{\mathrm{o}}$ " and " $\mathrm{X}_{\mathrm{t}}$ " indicate the concentration of AZM (in ppm) at time "zero" and " $\mathrm{t}$ ", respectively. Similarly, " $\mathrm{w}$ " and " $\mathrm{V}$ " and $\mathrm{m}$ are the total weight ( $\mathrm{g}$ ) or the mass of NE dispersed and the volume of prepared stock solution (mL), respectively (Assi et al. 2020).

\section{Morphological evaluation using transmission electron microscopy (TEM)}

The TEM tool is the most sophisticated technology to visualize nanoscale structures. Therefore, the TEM was used to visualize the architectural property of the optimized ANE5. The shape and size were analyzed using the TEM facility (Philips, Tecnai, Eindhoven, Netherlands). The sample was previously placed on the copper grid followed by negative staining with phosphotunsgtic acid $(0.1 \% \mathrm{w} / \mathrm{v})$. Excess sample was removed using an adsorbing filter paper. Then, the sample was visualized under TEM at 
various magnification. The size was also marked on an average area to compare with DLS results. The size was also estimated to get a fold error using equation 2 .

\section{Result And Discussion}

\section{Identification and solubility studies of AZM}

AZM is water insoluble drug having multiple therapeutic actions (bacteriostatic and bactericidal) against various bacterial infections. However, the drug is reported as one of the most potential pharmaceutical contaminants introduced into water through different sources. Several authors reported that conventional method is inefficient to remove from wastewater (influent and effluent samples) completely. This efficiency depends upon several factors such as physicochemical properties of $A Z M$, nature of water (effluents and sources) and method adopted for water treatments. Pharmaceutical drug concentration (as water pollutant) and risk to environmental burden is also associated with the population density and livestock unit (Osorio et al. 2016). The drug is chemically azalide derived from erythromycin which is a subclass of macrolide (figure $1 \mathrm{~A}$ ) with limited aqueous solubility $(0.0023 \mathrm{mg} / \mathrm{mL})$, high $\log P(4)$ and high pKa (8.5). AZM anhydrous is derived from hydrated AZM (AZM dihydrate) with slight difference in aqueous solubility $(0.514 \mathrm{mg} / \mathrm{mL}$ ) and $\log P(3.0)$ (Mutak, 2007). The result of DSC analysis confirmed anhydrous form of AZM as evidenced with the experimentally obtained value of melting point (fusion temperature) temperature $\left(115.8^{\circ} \mathrm{C}\right.$ ) (figure $\left.1 \mathrm{~B}\right)$. The drug solubility in water plays an important role for removal efficiency from bulk aqueous solution. Therefore, it was prerequisite to investigate AZM solubility in various oils, surfactant and co-surfactants for green nanoemulsion. The result showed that AZM was maximally soluble in capryol-90 (among oil), labrasol and THP (among surfactant) and ethanol (among co-surfactant) as shown in figure 2 . The experimentally obtained solubility of AZM in ethanol, capryol90 (MG90), THP, and labrasol were found as $11.0 \pm 0.55,78.9 \pm 3.95,83.5 \pm 4.2$, and $94.3 \pm 4.7 \mathrm{mg} / \mathrm{mL}$, respectively. The findings were in close agreement with previous reports where capryol-90 was used an excipient for nanoemulsion formulation (Assi et al. 2020). The obtained maximum solubility of AZM in MG90 and labrasol may be attributed to the lowest value of HLB (5 for MG90) and the hydrogen bond formed between the free H-O- groups of AZM and PEG (polyethylene glycol part of labrasol) [23]. Chemically, THP is 2-(2-ethoxyethoxy)ethane with one free hydroxyl functional group (O-H) which might have formed hydrogen bonding for improved solubility of AZM (Kauss et al. 2013). Ethanol solubilized the drug in considerable amount and selected as co-surfactant for nanoemulsion preparation.

\section{Pseudoternary phase diagrams and prepared nanoemulsions}

Several batches of water/THP/ethanol/MG-90 green nanoemulsions were prepared after selection of oil, surfactant and co-surfactants. The solubility study was a preliminary study to identify the most suitable excipients for green nanoemulsion. Pseudoternary phase diagrams dictated various possible nanoemulsions. Thus, MG90 (oil phase), THP (as surfactant), and ethanol (as co-surfactant) were finally screened based on maximum drug solubilization. Various nanoemulsions were tailored using different $S_{\text {mix }}$ ratio and the phase diagrams helped to suggest the most suitable $S_{\text {mix }}$ based on the maximum zone 
of nanoemulsion delineated. The phase diagrams were constructed by oil phase titration against each $S_{\text {mix }}$ to delineate water/THP/ethanol/MG90 green nanoemulsion (Shakeel et al. 2014c). As shown in figure 3 , the figure 3D showed that the $S_{\text {mix }}$ ratio "1:3" exhibited the maximum delineated zone of nanoemulsion. Therefore, this $\mathrm{S}_{\text {mix }}$ was used to prepare various formulations with varied concentration of MG90 and water as shown in table 1. The results obtained from phase diagrams suggested that nanoemulsion prepared with equal ratio of surfactant (THP) and co-surfactant (ethanol) $\left(S_{\text {mix }}=1: 1\right)$ was capable to solubilize only small concentration of water $(11.8 \% \mathrm{w} / \mathrm{w})$ using $40 \% \mathrm{w} / \mathrm{w}$ of $\mathrm{S}_{\text {mix }}$ (figure $3 \mathrm{~A}$ ). The highest concentration of water phase solubilized by the $S_{\text {mix }}$ ratio of $1: 2$ was approximately $13 \% \mathrm{w} / \mathrm{w}$. This was a slight increment in water solubilization by incorporating $50 \% \mathrm{w} / \mathrm{w}$ of $\mathrm{S}_{\text {mix }}$ (figure 3B). Moreover, when the concentration of co-surfactant (ethanol) with respect to surfactant (THP) was further increased $\left(60 \% \mathrm{w} / \mathrm{w}\right.$ at $\mathrm{S}_{\mathrm{mix}}$ ratio of 1:3), the maximum content of aqueous phase was found to be remarkably increased up to $28 \%$ (figure $3 \mathrm{C}$ ). In contrast, when the concentration of surfactant (THP) was relatively increased with respect to co-surfactant (ethanol), the dissolved concentration of water was decreased and subsequently the delineated zone was found to be reduced at $S_{\text {mix }}$ ratio of 2:1 (figure 3D). Thus, co-surfactant (ethanol) had significant impact to maximize water solubilization and expanded delineated zone in the phase diagrams. THP and capryol-90 were reported to be suitable components for green nanoemulsion synthesis and a suitable approach to remove hydrophobic pharmaceutical contaminant (diclofenac sodium) from a bulk aqueous solution (Ngan et al. 2014). However, the best optimized ratio of $S_{\text {mix }}(1: 3)$ was found to be 1:3 in our case which may be due to different physicochemical properties of AZM compared to diclofenac sodium. These were placed overnight to observe any sign of instability (benchtop stability). ANE1-ANE5 were prepared with varying concentration $(6-18 \% \mathrm{w} / \mathrm{w})$ of aqueous phase $\left(\mathrm{w} / \mathrm{o}\right.$, water in oil) at fixed concentration of $\mathrm{S}_{\text {mix }}(60 \% \mathrm{w} / \mathrm{w})$. The prepared five (ANE1-ANE5) nanoemulsions were subjected for further evaluations.

\section{Thermodynamic stability: Freeze thaw and centrifugation cycles}

The developed nanoemulsions were investigated for their thermodynamic stability by passing through various cycles of heating (thaw) and cooling steps followed by centrifugation. The results are presented in table 2. ANE1-ANE5 were found to be stable and exhibited no signs of any physical instability under stress condition of temperatures and centrifugation. Notably, all of the nanoemlsions maintained homogeneous state after removing from tested temperature to room temperature. It is good to correlate the functionality of $\mathrm{S}_{\mathrm{mix}}$ for improved stability against temperature. The surfactant and co-surfactant combination was in right ratio and concentration to prevent the disruption of lipid film surrounding the water droplets in nanoemulsion (Pal, 2016). It means the developed green nanoemulsions are stable and suitable to store at all explored temperatures.

\section{Evaluation parameters of green nanoemulsions}

Prepared nanoemulsions were characterized for globular size, zeta potential, PDI, viscosity, and RI values. The results are summarized in table 3 . It is apparent that all of the composition of nanoemulsions 
(ANE1-ANE5) were stable with the size range of $49-149 \mathrm{~nm}$, PDI value of 0.17-0.34, zeta potential range from -27.8 to $-34.4 \mathrm{mV}$, and viscosity range of 92.8-162.9 cP. ANE5 and ANE1 showed the lowest (49 nm) and the highest ( $149 \mathrm{~nm}$ ) values of globular size, respectively. Moreover, the globule size of nanoemulsion (ANE1-ANE5) was found to be decreased with increasing concentration of aqueous phase (water) whereas this was constantly increased with increasing content of MG-90 (organic oil phase) (table 3). The lowest value of globule in ANE5 was probably due to the lowest content of MG-90 and the maximum content of aqueous phase containing surfactant. An effect of the aqueous phase and organic lipid phase on the globular size of water/ethanol/transcutol/MG90 nanoemulsion was portrayed in figure 4A. The observed trend was in good agreement with previous report wherein transcutol and capryol based green nanoenulsion revealed remarkable impact of the water and oil contents on particle size in similar pattern (Shakeel et al. 2014c).

It was also important to correlate the impact of the aqueous and organic phase of water/ethanol/transcutol/MG90 nanoemulsion on rheological behavior such as viscosity. It is well established fact that viscosity of any liquids depends upon several factors such as temperature, applied force, structural break down, composition, dipole strength of droplets, size, and shape of droplets (Shakeel et al. 2013). In general, the viscosity of water in oil type of nanoemulsion (w/o) elicits greater viscosity as compared to oil in water type of nanoemulsion $(\mathrm{o} / \mathrm{w})$ which is quite obvious due to higher content of oil in former nanoemulsion. Moreover, the relative viscosity of o/w type of nanoemulsion is strongly influenced with the solvation of globules in continuous phase (Shakeel et al. 2013). It is one of the most key thermophysical properties of nanoemulsions required for the design, selection, and operation of an equipment engaged for handling, mixing, formulating, storage, processing, and pumping of nanoemulsions (Shakeel et al. 2013). In the present study, we investigated the impact of water and MG-90 contents on the viscosity of nanoemulsions (ANE1-ANE5). Result has been presented in table 3 . The highest (162.9 cP) and the lowest (92.8 cP) values of viscosity were observed for ANE1 and ANE5, respectively. This may be prudent to correlate with the highest content of MG-90 oil in ANE1 and vice versa. In this study, we carried out all analysis at constant temperature throughout the experiment. We found that the viscosity of nanoemulsions (ANE1-ANE5) was quite decreasing with decrease in globule size and the concentration MG-90 oil whereas the viscosity was found to be decreased with increased concentration of water. The impact of both phases on the viscosity of water/ethanol/transcutol/MG-90 nanoemulsion has been illustrated in figure 4B. The finding is in accordance with the previous report where hydrophilic 5-fluorouracil was formulated in w/o type of nanoemulsion (Alliod et al. 2019). The values of PDI of water/ethanol/transcutol/MG-90 nanoemlsions (ANE1-ANE5) were found to be in the range of 0.17-0.34. The maximum value of PDI was observed in ANE1 (0.34) whereas this was minimum in ANE5 (0.17) suggesting homogeneous nature of developed nanoemulsions (table 3 ). The impact of aqueous (water) and organic phase (MG-90) on PDI is illustrated in figure 4C. Notably, the value of PDI was found to be decreased from ANE1 to ANE5 due to decreased content of MG-90 and increased concentration of water. The ANE5 could be considered as one of the most stable, homogeneous and quite consistent green nanoemulsion among them. This may be opted for improved removal efficiency of AZM from aqueous solution providing substantial exposed surface area. 
The values of observed refractive index of nanoemulsions were found to be in the range of 1.33111.3488 (ANE1-ANE5) as shown in table 3. The maximum value (1.3488) was associated with ANE1 which may probably be due to the highest content of MG-90. The values of RIs were constantly decreasing with increased content of water from ANE1 to ANE5. Refractive index depends upon several factors. It is an optical property of nanmoemulsion which represents transparent behavior of solution or nanoemulsion with the lowest globular size. The values of RI for water and MG-90 were 1.33 and 1.35, respectively. The RI value approaching close to 1.33 (RI of water) indicates isotropic, transparent and thermodynamically stable nanoemulsion. These values may also be correlated with the size. RI values decrease with decrease in size of globule in nanoemulsion due to maximum refraction and least chances of scattering of light. The globule size of nanoemulsion approaching near or beyond the wavelength of visible spectrum causes scattering of light and abbe type of refractometer is not valid instrument.

Zeta potential indicates the surface charge density of globules dispersed in continuous phase of nanoemulsion. The negative values of zeta was attributed to MG-90 medium chain tryglycerides. The values of zeta potential are presented in table 3 and these values were found to be decreased with decrease in concentration of MG-90 oil in nanoemulsion. The values of zeta potential of (water/ethanol/transcutol/MG-90) ANE1, ANE2, ANE3, ANE4, and ANE5 were -34.4, -32.3, -30.7, -29.7, and $-27.8 \mathrm{mV}$, respectively. These values supported the finding of thermodynamic stability testing where all nanoemulsions passed series of heating and cooling cycles of thermodynamic stress test. Nanoemulsion possessing zeta potential value nearly $\pm 30 \mathrm{mV}$ was considered as stable system reported in several literatures (Pal 2016; Shakeel et al. 2013; Shakeel et al. 2014c).

\section{Adsorption study: percent removal efficiency (\%RE)}

As we discussed earlier that AZM is a critical pharmaceutical contaminant introduced in aquatic system from various sources as effluents (wastewater, municipal effluent, industrial, and hospital effluents). The process of removal of the drug dissolved in aquatic system depends upon several factors such as physicochemical properties of the drug, adsorbent, and processing parameters (Alliod et al. 2019). The removal of trace content of AZM from water is a critical and difficult task due to poor aqueous solubility, poor adsorption, and conventional treatment plant (time consuming and expensive). Therefore, it was necessary to investigate the removal efficiency of water/ethanol/transcutol/MG-90 nanoemulsions (ANE1-ANE5) from aqueous system containing well known antibiotic "AZM". Results are exhibited in figure 5 , figure 6 and 7.

The time of exposure of water/ethanol/transcutol/MG-90 nanoemulsion may have impact on the \%RE of AZM from a bulk aqueous solution. Therefore, we also investigated the impact of contact or exposure time of nanoemulsion at three different time point such as 5, 10, and $20 \mathrm{~min}$. The results of \%RE of water/ethanol/transcutol/ MG-90 nanoemulsions (ANE1-ANE5) are presented in table 4. There was insignificant impact of the "exposure time" on \%RE of nanoemulsions (ANE1-ANE5) from aqueous solution of AZM. The result of \% AZM adsorption onto the surface of water/ethanol/transcutol/MG-90 nanoemulsions (ANE1-ANE5) is summarized in table 4. The \%RE of the nanoemulsions (ANE1-ANE5) was 
found to be in the range of $72.7-96.8 \%$ (table 4). Interestingly, there was no significant change in percent AZM adsorption with varied exposure time of nanoemulsions with aqueous solution on $\% \mathrm{RE}$ of AZM which may be due to negligible impact of contact time on \% RE of AZM from the aqueous solution (figure 7). The nanoemulsion ANE1 and ANE5 exhibited the lowest (72.7\%) and the highest (96.8\%) values of \%RE of AZM from an aqueous bulk solution at the end of 20 min of contact time (exposure time), respectively. Removal of pharmaceutical contaminant from an aqueous solution depends upon several factors such as dispersed phase, dispersing phase, globule size, viscosity of dispersed and dispersing phase of nanoemulsions (o/w and w/o), surfactants, and processing variables (Alliod et al. 2019; Shakeel et al. 2014a; Shakeel et al. 2014c; Shakeel et al. 2015). Therefore, the impact of water (aqueous phase) and MG90 (lipid organic phase) was investigated which has been illustrated in figure 5 . Notably, when the content of MG90 was increased (from 22\% to $34 \%$ ) and the content of water was decreased (from $18 \%$ to $6 \%$ ), the \%RE was found to be decreased rapidly as shown in figure 5 . Thus, both primary components of water/ethanol/transcutol/MG90 green nanoemulsion (ANE1-ANE5) had significant effect of the \% RE of AZM from aqueous bulk solution. Removal of AZM from an aqueous solution is based on adsorption mechanism onto the available nanoglobules of MG90 (Shaeel et al. 2014b; Shakeel et al. 2015]. The surface area depends upon the size of globules. Therefore, it may be prudent to correlate the \% RE of nanoemulsions with the result of globule size and viscosity. The result showed that the \%RE was found to be increased with decrease in globule size and viscosity of water/ethanol/transcutol/MG90 nanoemulsions (ANE1-ANE5) (figure 6A-B). The maximum \%RE of AZM was observed with ANE5 (96.8\% at $20 \mathrm{~min}$ ) possessing the globular size of $\sim 93 \mathrm{~nm}$ and the viscosity of $1.334 \mathrm{cP}$. Moreover, the lowest value of \%RE (71.5\% at $5 \mathrm{~min})$ of AZM was found to be with ANE1 possessing the globular size of $149 \mathrm{~nm}$ and the viscosity of $162.9 \mathrm{cP}$. These findings are in agreement with the previous report where authors explored capryol-90 based green nanoemulsion to remove lipophilic drug contaminants from aqueous bulk solution [Shakeel et al. 2014a; Shakeel et al. 2014c; Shakeel et al. 2015]. Figure 8A-B illustrated the globule size intensity and TEM scanned image of the best ANE5. The globule of water/ethanol/transcutol/MG90 nanoemulsion ANE5 was spherical in shape and the size was in supportive to the results of DLS based globule size. However, there was a slight variation in size obtained from both techniques. Therefore, a fold error was calculated which was found to be 1.35 which is under acceptable range $(<2.0)$.

\section{Proposed mechanism}

Green nanoemulsions are thermodynamically stable and isotropic system with efficient stability against dilution with continuous phase. The w/o nanoemulsion get converted to o/w type of nanoemulsion upon excess dilution with dispersed phase (aqueous). This happens when w/o type of green nanoemulsion is exposed with higher volume of water. In case of aqueous drug solution containing low concentration of hydrophobic drug, insoluble drug starts to precipitate upon dilution while nanoemulsion conversion and relative diffusion of AZM between water and oil. Thus, drug is precipitated due to water insolubility when AZM is transported from aqueous solution towards green nanoemulsion phase. A drug precipitation through insolubility could be every possible mechanism to remove AZM from bulk aqueous solution. This 
rate of removal may be influenced with the content of water, MG90, globule size, viscosity and other factors. An illustrative schematic image of proposed mechanism has been portrayed in figure 9 .

\section{Conclusion}

The study addressed to develop water/ethanol/transcutol/capryol-90 based green nanoemulsion for adsorptive removal of AZM from contaminated aqueous system at room temperature. Excipients were selected based on the drug solubility followed by nanoemulsions as dictated by phase diagrams. The globular size, PDI, viscosity, zeta potential, refractive index and \% RE were influenced with the content of water and oil in the nanoemulsion. Based on the lowest value of size, PDI, highest zeta potential, maximum \%RE, ANE5 was considered as the most robust and optimum green nanoemulsion to remove AZM from an aqueous solution. Thus, ANE5 may be suitable nanoemulsion for lipid adsorption based adsorptive removal of AZM from aqueous solution. The approach is simple, economic, scalable, efficient, and ecofriendly as compared to conventional methods.

\section{Declarations}

\section{Ethical approval}

The study does not involve any animal or animal related experiments.

\section{Consent to participate}

All authors agreed to participate in the present research

\section{Consent to Publish}

All authors agreed to publish this research finding

\section{Authors contribution statement}

Afzal Hussain: Conceptualization, drafting and writing, Sultan Alshehri: Data curation, Mohammad A. Altamimi: Software, data curation and formal review; Mohd. Usman Mohd Siddique: Software and analysis

\section{Acknowledgement}

The authors would like to thank the Deanship of Scientific Research at King Saud University for supporting this study through research group project number RG-1441-462.

\section{Conflict of interest}

Authors report no conflict of interest 


\section{Funding}

The authors would like to thank the Deanship of Scientific Research at King Saud University for supporting this study through research group project number RG-1441-462

\section{References}

Ali MS, Alam MS, Alam N, Siddiqui MR (2014) Preparation, Characterization and Stability Study of Dutasteride Loaded Nanoemulsion for Treatment of Benign Prostatic Hypertrophy. Iranian Journal of Pharmaceutical Research 13 (4): 1125-1140.

Alliod O, Messager L, Fessi H, Dupin D, Charcosset C (2019) Influence of viscosity for oil-in-water and water-in-oil nanoemulsions production by SPG premix membrane emulsification. Chemical Engineering Research and Design, Elsevier 142: 87-99.doi.10.1016/j.cherd.2018.11.027.

Assi RA, Abdulbaqi IM, Ming TS, Yee CS, Wahab HA, Asif SM, Darwis Y (2020) Liquid and Solid SelfEmulsifying Drug Delivery Systems (SEDDs) as Carriers for the Oral Delivery of Azithromycin: Optimization, In Vitro Characterization and Stability Assessment. Pharmaceutics 12(11):1052; https://doi.org/10.3390/pharmaceutics12111052.

Ávila C, García-Galán MJ, Borrego CM, Rodríguez-Mozaz S, García J, Barceló D (2021) New insights on the combined removal of antibiotics and ARGs in urban wastewater through the use of two configurations of vertical subsurface flow constructed wetlands. Science of The Total Environment 755, 142554. doi:10.1016/j.scitotenv.2020.142554.

CECs in the Watch List of Commission Decision 2015/495/EU.

Davoodi S, Dahrazma B, Goudarzi N, Gorji HG (2019) Adsorptive removal of azithromycin from aqueous solutions using raw and saponin-modified nano diatomite. Water Sci Technol 80(5): 939-949. doi: 10.2166/wst.2019.337.

Fent K, Weston AA, Caminada D (2006) Ecotoxicology of human pharmaceuticals. Aquatic Toxicology 76 (2): 122-159.

Ghosh GC, Hanamoto S, Yamashita N, Huang X, Tanaka H (2016) Antibiotics Removal in Biological Sewage Treatment Plants. Pollution 2(2): 131-139.

Hussain A, Singh SK, Singh N, Verma, PRP (2016) In vitro-in vivo-in silico simulation studies of antitubercular drugs doped with a self-nanoemulsifying drug delivery system. RSC Advances 6(95): 9314793161.

Kauss T, Gaubert A, Boyer C, Ba BB, Manse M, Massip S, Jean-Michel L, Fawaz F, Lembege M, JeanMichel B, Lafarge X, Lindegardh N, White NJ, Olliaro P, Millet P, Gaudin K (2013) Pharmaceutical 
development and optimization of azithromycin suppository for paediatric use. International Journal of Pharmaceutics 441(1-2): 218-226.

Khalil AME, Memon FA, Tabish TA, Fenton B, Salmon D, Zhang S, Butler D (2021) Performance Evaluation of Porous Graphene as Filter Media for the Removal of Pharmaceutical/Emerging Contaminants from Water and Wastewater. Nanomaterials 11: 79. https://doi.org/10.3390/nano11010079.

Luo X, Hao T, Yue L, Hong G, Lu Y (2016) Azithromycin Wastewater Treatment with La Doping Titanium Dioxide / Active Carbon Composites. 4th International Conference on Sensors, Measurement and Intelligent Materials (ICSMIM 2015): 861-870.

Luo Z, Yu G, Han X, Yang T, Ji Y, Huang H, Wang G, Liu Y, Sun W (2020) Prediction of the pharmacokinetics and pharmacodynamics of topiroxostat in humans by integrating the physiologically based pharmacokinetic model with the drug-target residence time model. Biomedicine \& Pharmacotherapy 121: 109660. doi:10.1016/j.biopha.2019.109660.

Mackul'ak T, Nagyová K, Faberová M, Grabic R, Koba O, Gál M, Birošová L (2015) Utilization of Fenton-like reaction for antibiotics and resistant bacteria elimination in different parts of WWTP. Environmental Toxicology and Pharmacology 40(2): 492-497.

Mahdi WA, Hussain A, Bukhari SI, Alshehri S, Singh B, Ali N (2021) Removal of clarithromycin from aqueous solution using water/triton X-100/ ethanol/ olive oil green nanoemulsion method. Journal of Water Process Engineering 40: 101973. doi.org/10.1016/j.jwpe.2021.101973.

Muñoz-Calderón A, Zúñiga-Benítez H, Valencia SH, Rubio-Clemente A, Upegui SA, Peñuela GA (2020) Use of low frequency ultrasound for water treatment: Data on azithromycin removal. Data in Brief 31: 105947. doi:10.1016/j.dib.2020.105947.

Mutak S (2007) Azalides from azithromycin to new azalide derivatives. The Journal of Antibiotics 60(2): $85-122$.

Ngan CL, Basri M, Tripathy M, Karjiban AR, Abdul-Malek E (2014) Physicochemical Characterization and Thermodynamic Studies of Nanoemulsion-Based Transdermal Delivery System for Fullerene. The Scientific World Journal: 1-12 doi:10.1155/2014/219035.

Osorio V, Larrañaga A, Aceña J, Pérez S, Barceló D (2016) Concentration and risk of pharmaceuticals in freshwater systems are related to the population density and the livestock units in Iberian Rivers. Science of The Total Environment 540: 267-277.

Pal R (2016) Modeling the Viscosity of Concentrated Nanoemulsions and Nanosuspensions. Fluids 1(2): 11. doi:10.3390/fluids1020011.

Segura PA, Francois M, Gagnon C, Sauve S (2009) Review of the occurrence of anti-infectives in contaminated wastewaters and natural and drinking waters. Environmental Health Perspectives 117 (5): 
Shakeel F, Haq N, Ahmed MA, Gambhir D, Alanazi FK, Alsarra IA (2014) Removal of diclofenac sodium from aqueous solution using water/Transcutol/ethylene glycol/Capryol-90 green nanoemulsions. Journal of Molecular Liquids 199: 102-107.

Shakeel F, Haq N, Alanazi FK, Alsarra IA (2014) Box-Behnken statistical design for removal of methylene blue from its aqueous solution using SDS self-microemulsifying systems. Ind Eng Chem Res 53 (3): 1179-1188.

Shakeel F, Haq N, Alanazi FK, Alsarra IA (2015) Removal of glibenclamide from aqueous solution using water/PEG-400/ethanol/eucalyptus oil green nanoemulsions. Journal of Molecular Liquids 203: 120124.

Shakeel F, Haq N, Al-Dhfyan A, Alanazi FK, Alsarra IA (2013) Chemoprevention of skin cancer using low HLB surfactant nanoemulsion of 5-fluorouracil: a preliminary study. Drug Delivery 22(4): 573-580.

Shakeel F, Haq N, Sumague TS, Alanazi FK, Alsarra IA (2014) Removal of indomethacin from aqueous solution using multicomponent green nanoemulsions. Journal of Molecular Liquids 198: 329-333.

Verlicchi P, Zambello E (2014) Howefficient are constructedwetlands in removing pharmaceuticals from untreated and treated urban wastewaters? A review Sci Total Environ 470-471: 1281-1306.

Zhu X, Fryd MM, Huangz JR, Mason TG (2012) Optically probing nanoemulsion compositions. Phys Chem Chem Phys 14: 2455-2461.

\section{Tables}

Table 1: Basic composition of selected (water/ethanol/transcutol/MG90) green nanoemulsions

\begin{tabular}{lllccc}
\hline Code & \multicolumn{6}{l}{ Composition $(\%$ w $/$ ) } \\
\cline { 2 - 6 } & Water & THP & Ethanol & MG90 & $\mathrm{S}_{\text {mix }}$ \\
\hline ANE1 & 6 & 15 & 45 & 34 & $1: 3$ \\
ANE2 & 9 & 15 & 45 & 31 & $1: 3$ \\
ANE3 & 12 & 15 & 45 & 28 & $1: 3$ \\
ANE4 & 15 & 15 & 45 & 25 & $1: 3$ \\
ANE5 & 18 & 15 & 45 & 22 & $1: 3$ \\
\hline
\end{tabular}

Note: MG90 = Capryol-90

Table 2: Freeze thaw cycles and centrifugation of thermodynamic stability study for green nanoemulsions (water/ethanol/transcutol/MG90) 


\begin{tabular}{|c|c|c|c|c|c|}
\hline \multirow[t]{2}{*}{ Code } & \multicolumn{5}{|c|}{ Thermodynamic stability cycles } \\
\hline & Centrifugation & Thaw $\left(45^{\circ} \mathrm{C}\right)$ & Freeze $\left(-21^{\circ} \mathrm{C}\right)$ & Cooling $\left(4^{\circ} \mathrm{C}\right)$ & $\mathrm{S}_{\operatorname{mix}}$ \\
\hline ANE1 & $\sqrt{ }$ & $\sqrt{ }$ & $\sqrt{ }$ & $\sqrt{ }$ & $1: 3$ \\
\hline ANE2 & $\sqrt{ }$ & $\sqrt{ }$ & $\sqrt{ }$ & $\sqrt{ }$ & $1: 3$ \\
\hline ANE3 & $\sqrt{ }$ & $\sqrt{ }$ & $\sqrt{ }$ & $\sqrt{ }$ & $1: 3$ \\
\hline ANE4 & $\sqrt{ }$ & $\sqrt{ }$ & $\sqrt{ }$ & $\sqrt{ }$ & $1: 3$ \\
\hline ANE5 & $\sqrt{ }$ & $\sqrt{ }$ & $\sqrt{ }$ & $\sqrt{ }$ & $1: 3$ \\
\hline
\end{tabular}

Note: $\sqrt{ }$ mark indicates passed and there was no sign of physical instability.

Table 3: Evaluated parameters of nanoemulsions (water/ethanol/transcutol/MG90)

\begin{tabular}{lllccl}
\hline Code & \multicolumn{5}{l}{ Results $(\mathrm{mean} \pm$ standard deviation) } \\
\cline { 2 - 6 } & Size $(\mathrm{nm})$ & PDI & $\eta(\mathrm{cP})$ & Refractive index & $\zeta(\mathrm{mV})$ \\
\hline ANE1 & $149.0 \pm 7$ & 0.34 & $162.9 \pm 4.4$ & $1.3488 \pm 0.002$ & $-34.4 \pm 3.5$ \\
ANE2 & $111.0 \pm 6$ & 0.31 & $154.2 \pm 4.1$ & $1.3478 \pm 0.003$ & $-32.3 \pm 3.4$ \\
ANE3 & $89.0 \pm 6$ & 0.23 & $141.9 \pm 3.8$ & $1.3469 \pm 0.002$ & $-30.7 \pm 3.0$ \\
ANE4 & $67.0 \pm 5$ & 0.20 & $132.7 \pm 3.1$ & $1.3358 \pm 0.002$ & $-29.7 \pm 2.6$ \\
ANE5 & $49.0 \pm 4$ & 0.17 & $92.8 \pm 2.8$ & $1.3341 \pm 0.001$ & $-27.8 \pm 2.5$ \\
\hline
\end{tabular}

Note: $\mathrm{PDI}=$ Polydispersity index, MG90 $=$ Miglyol-90, $\zeta=$ Zeta potential, and $\eta=$ Viscosity

Table 4: Percent removal efficiency of various nanoemulsion (water/ethanol/transcutol/MG90) at different time points

\begin{tabular}{lccc}
\hline \multirow{2}{*}{ NE code } & \multicolumn{2}{c}{ Percent removal efficiency $(\% \mathrm{RE})$} \\
\cline { 2 - 4 } & $5 \mathrm{~min}$ & \multicolumn{1}{c}{$10 \mathrm{~min}$} & $20 \mathrm{~min}$ \\
\hline ANE-5 & $95.8 \pm 3.1$ & $96.1 \pm 3.2$ & $96.8 \pm 3.5$ \\
ANE-4 & $90.3 \pm 2.9$ & $90.8 \pm 3.3$ & $91.2 \pm 3.4$ \\
ANE-3 & $84.7 \pm 2.5$ & $85.9 \pm 2.6$ & $86.6 \pm 2.9$ \\
ANE-2 & $79.8 \pm 1.9$ & $80.1 \pm 2.2$ & $80.8 \pm 2.1$ \\
ANE-1 & $71.5 \pm 2.3$ & $71.8 \pm 2.4$ & $72.7 \pm 2.7$ \\
\hline
\end{tabular}

Note: \%RE $=$ Percent removal efficiency

\section{Figures}




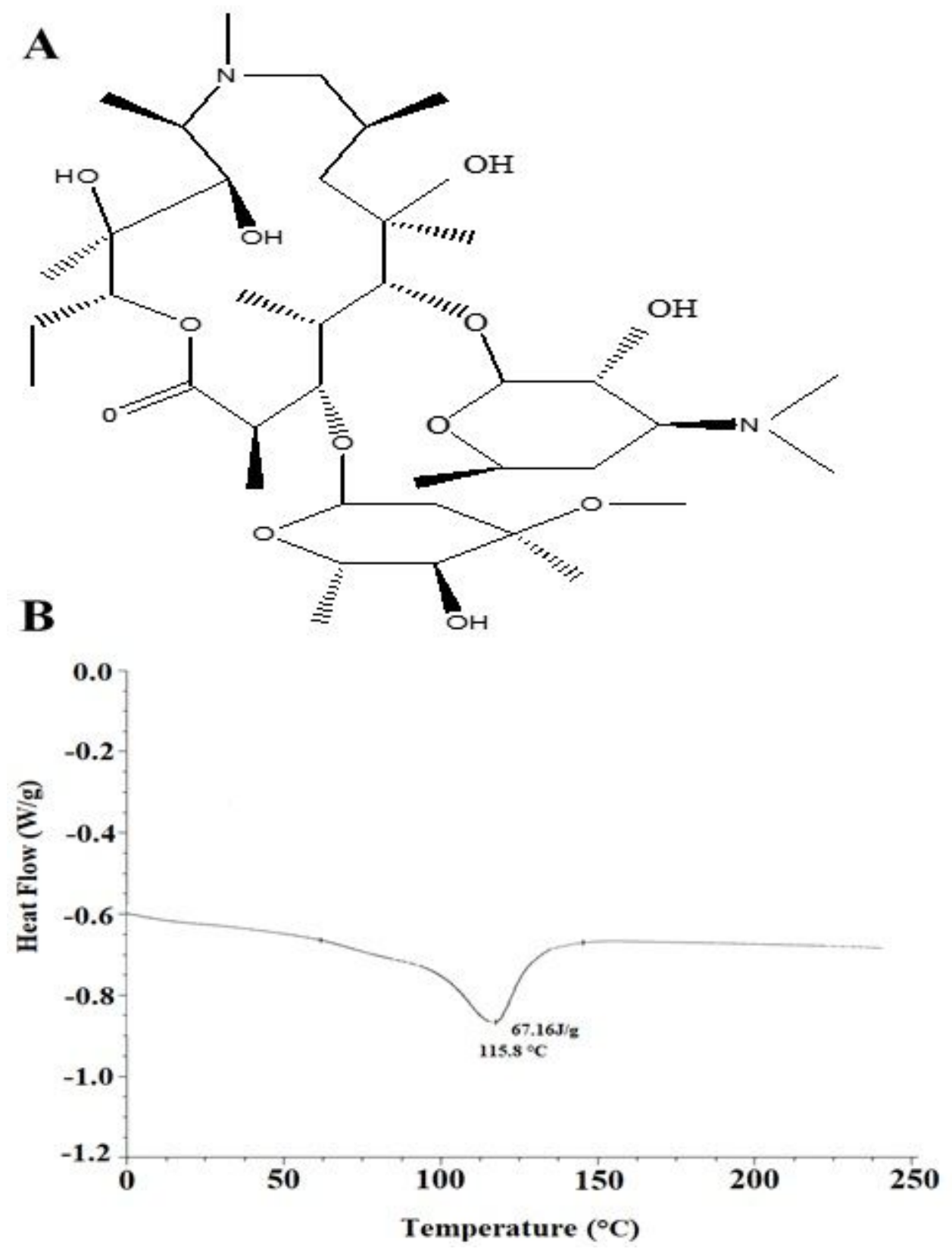

Figure 1

Chemical structure of azithromycin (A), and a DSC thermogram of neat AZM (B) 


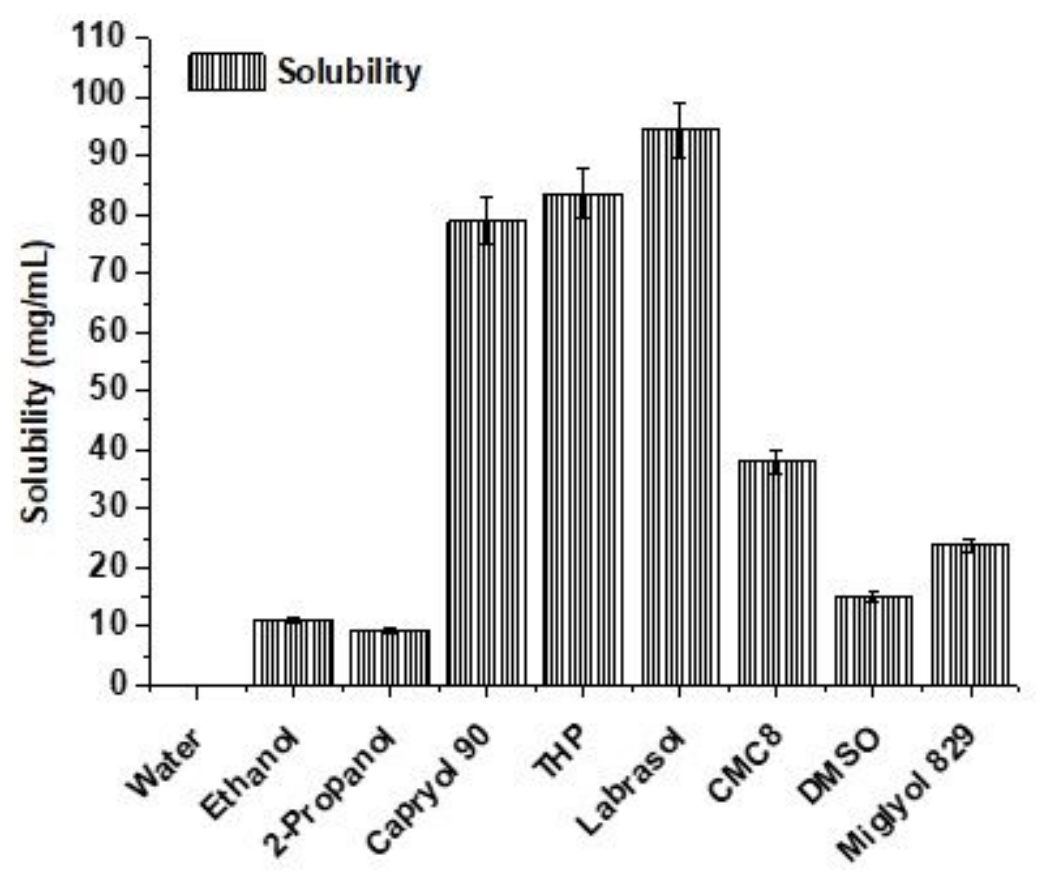

Figure 2

Solubility study of CIP in various solvents, surfactants, co-surfactants and lipids
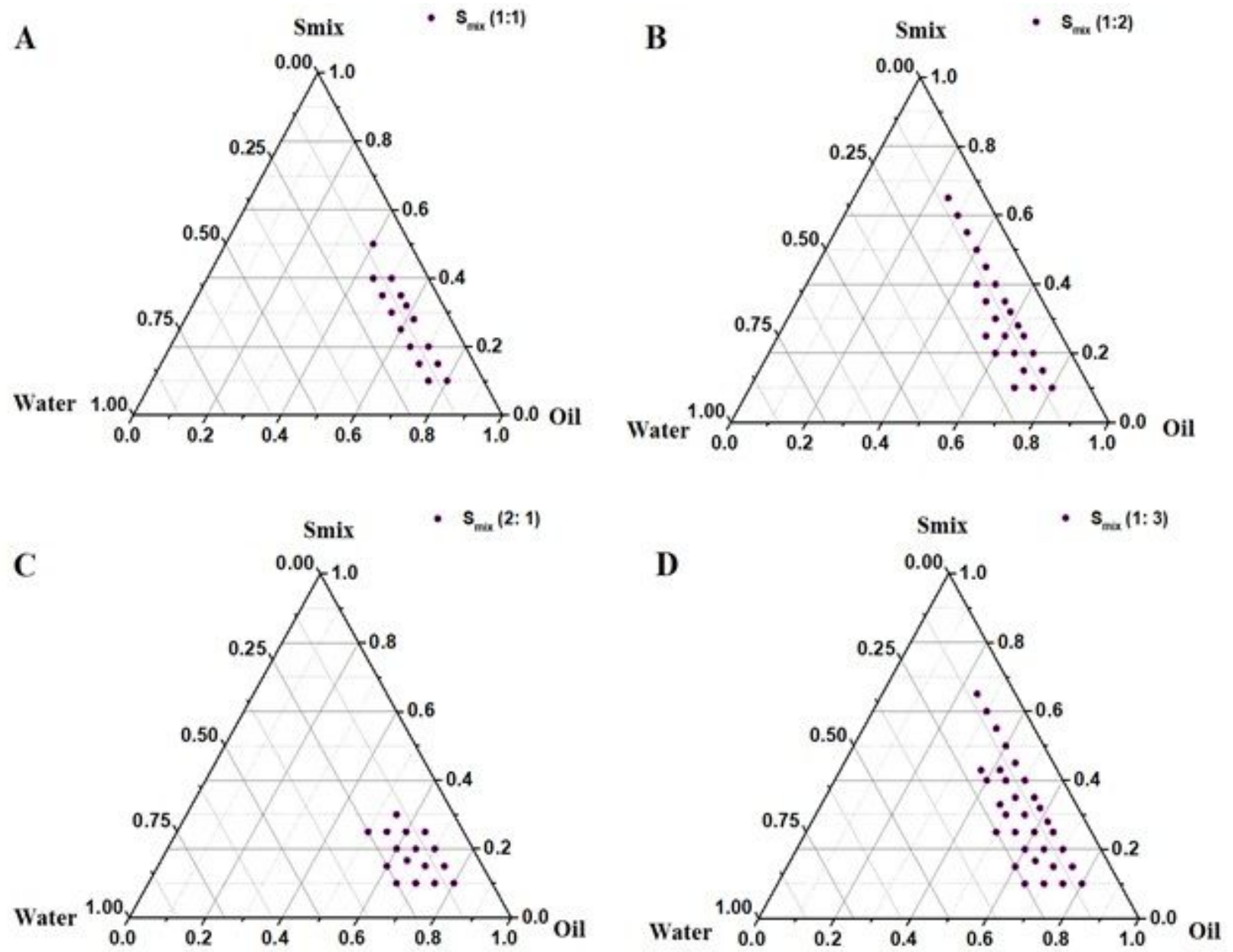

Figure 3 
Pseudo ternary phase diagram of azithromycin loaded nanoemulsions with varied Smix ratios.
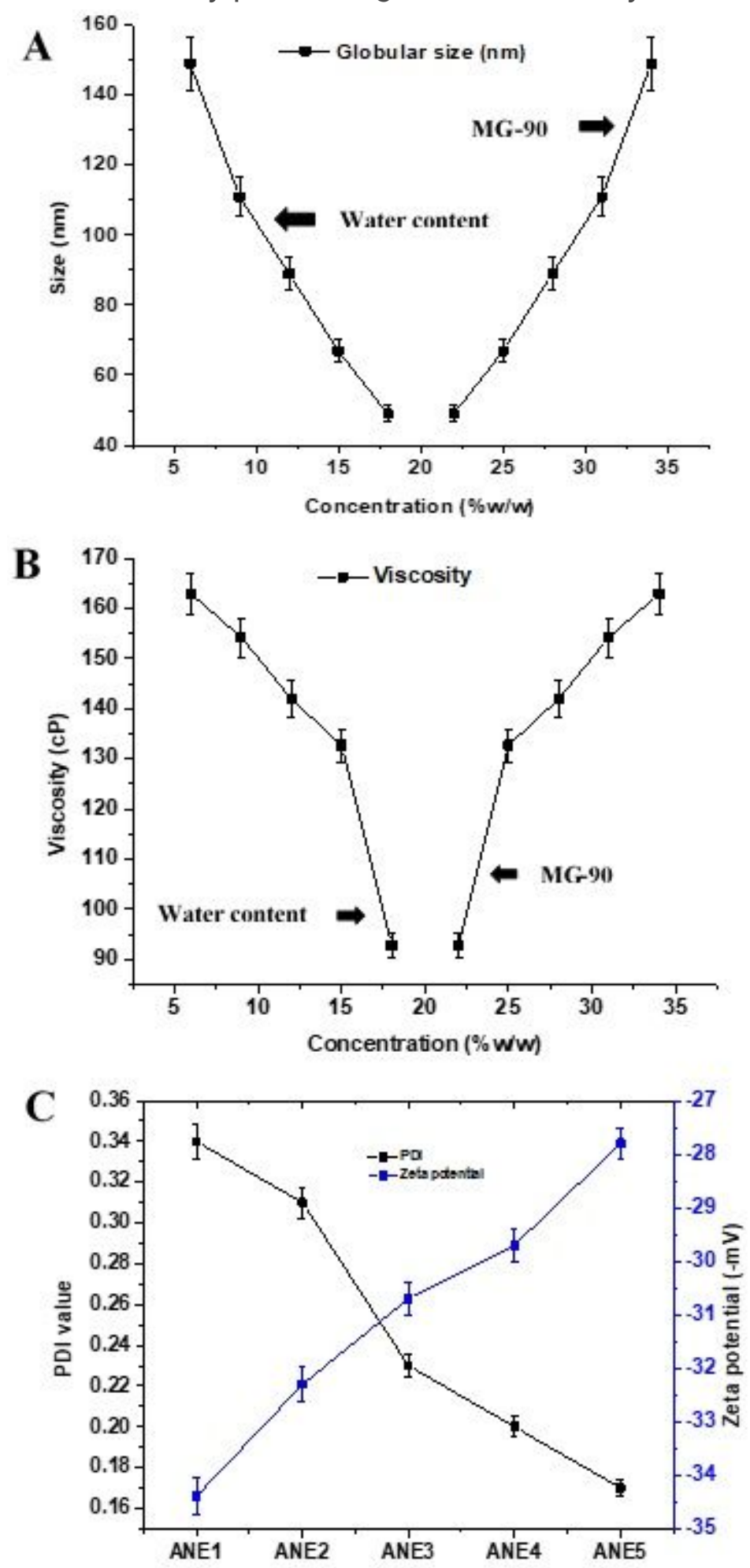

\section{Figure 4}

(A) Impact of the content of water and MG-90 on globular size of water/ethanol/transcutol/MG90 nanoemulsions, (B) Impact of MG-90 and water on viscosity (cP), (C) Polydispersity index and zeta potential of nanoemulsions (ANE1-ANE5). 


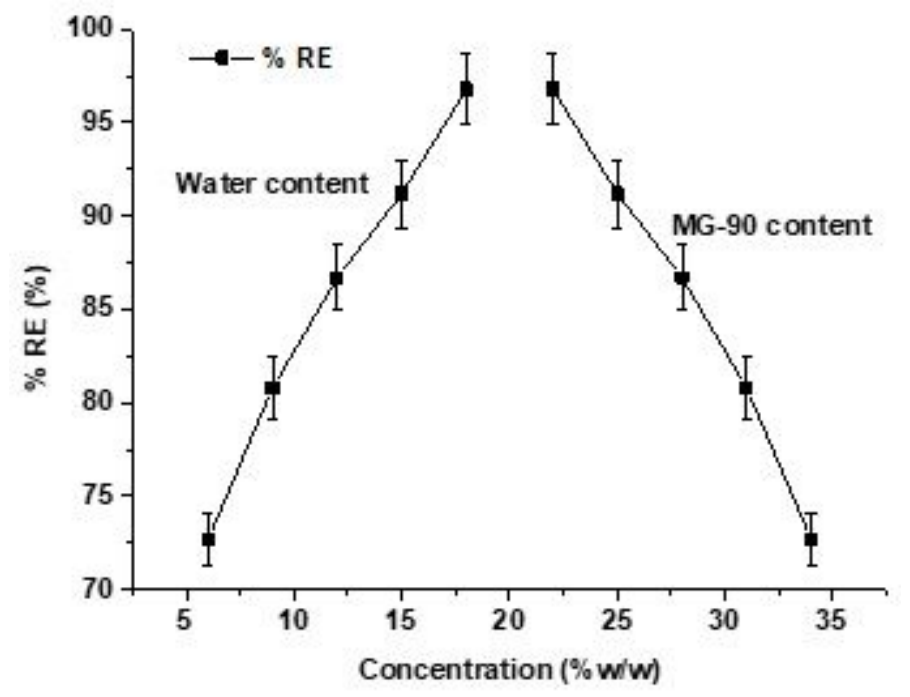

Figure 5

Impact of MG-90 and aqueous phase content on percent removal efficiency (\%RE) of AZM a bulk aqueous solution using green (water/ethanol/transcutol/ MG-90) nanoemulsions.
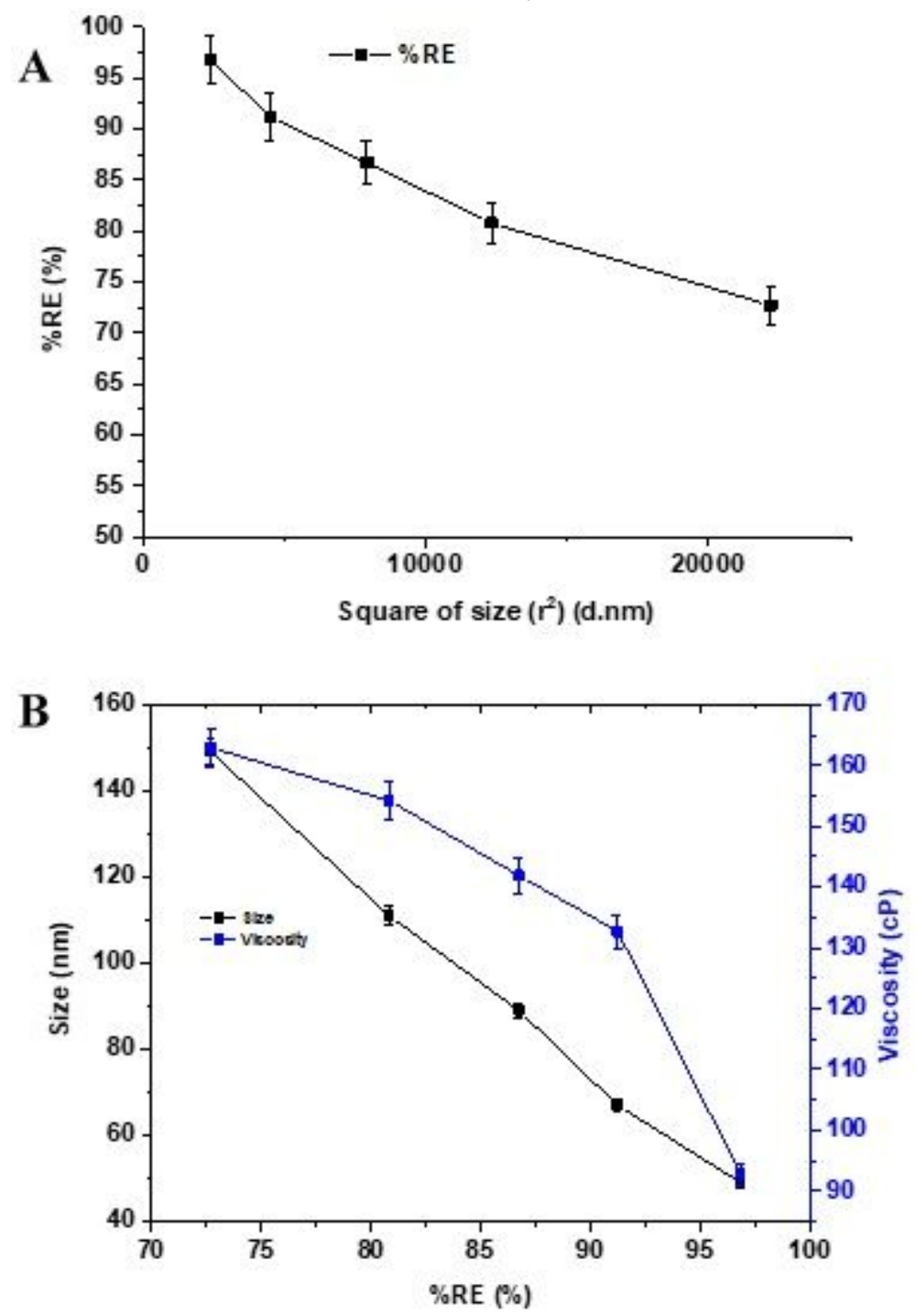
Figure 6

Impact of the square of size (r2) on \%RE of AZM by nanoemulsions (ANE1-ANE5)

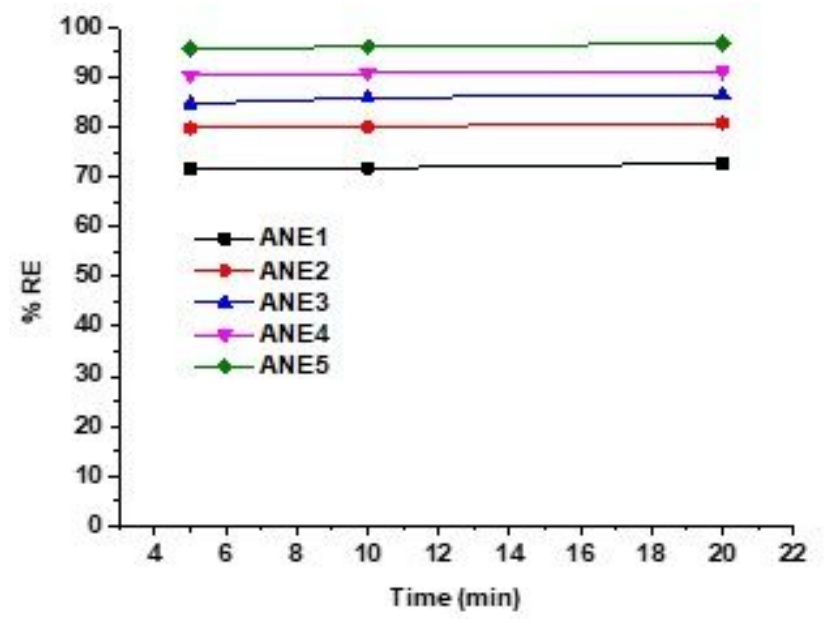

\section{Figure 7}

Impact of exposure time (min) on \%RE of AZM by nanoemulsions (ANE1-ANE5)
A
Size Distribution by Intensity

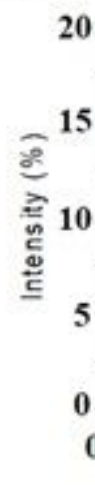

20
15
5
0
0.
0

-

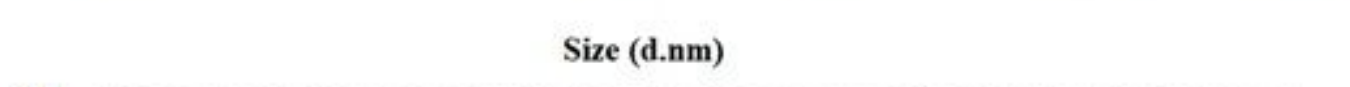

B

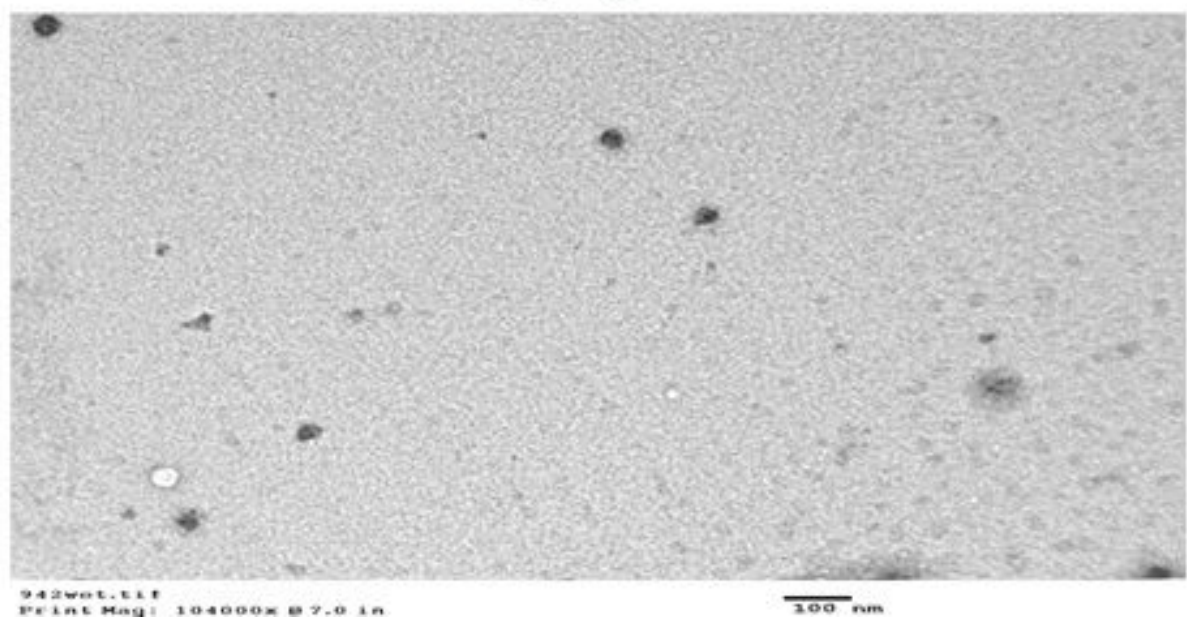

Figure 8 
Evaluation of optimized ANE5 green nanoemulsion: (A) globular size intensity, (B) transmission electron microscopy (TEM) photograph of ANE5 after dispersing in aqueous, and (C) TEM image of ANE5 loaded with AZM after dispersion of water/ethanol/transcutol/MG90 nanoemulsion with aqueous solution (magnification at 20000X).

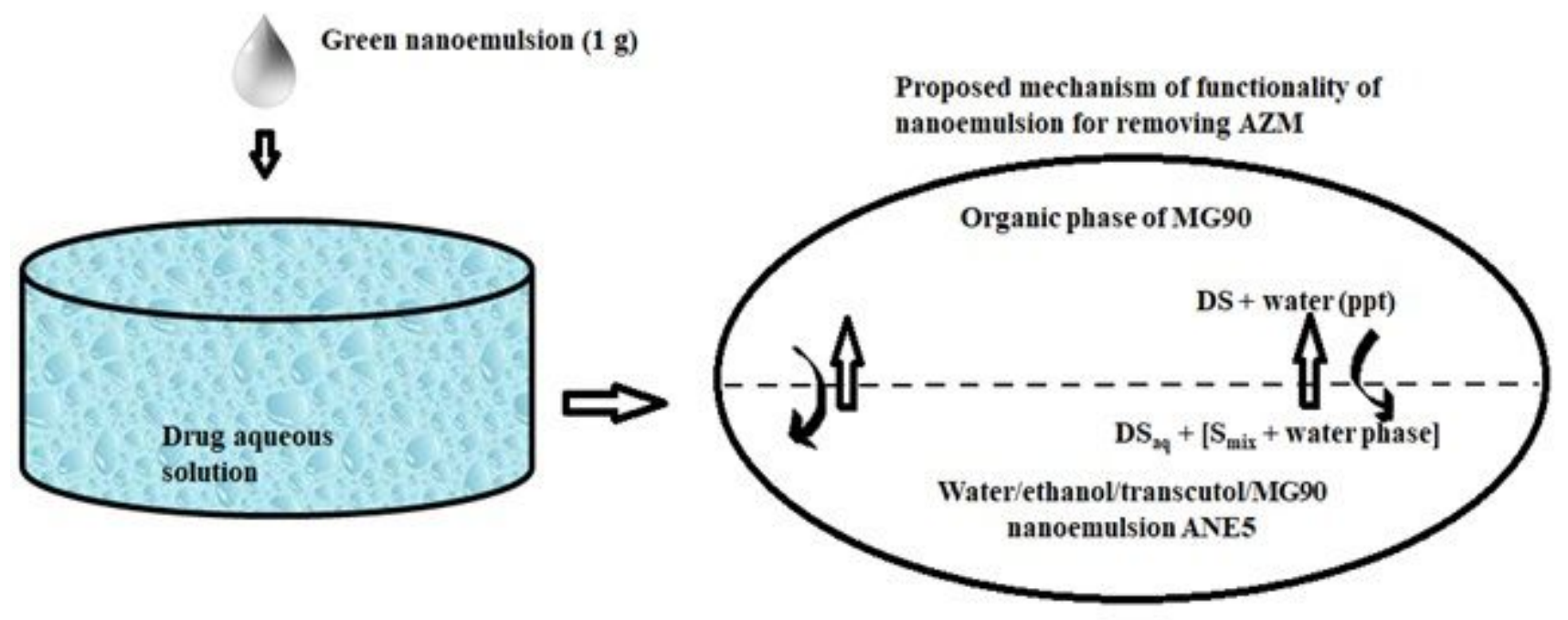

Figure 9

Proposed mechanism of ANE5 functionality to remove AZM from a bulk aqueous solution

\section{Supplementary Files}

This is a list of supplementary files associated with this preprint. Click to download.

- GraphicalAbstract.jpg 